normals on the curve representing the cranial isotope content. This peak is attributed to the failure of Antipyrine passing through the shunt to equilibrate with brain water, as normally occurs upon passage through the brain capillary bed. This initial peak representing the A-V shunt is aggravated following hyperventilation. This might be secondary to the shift of blood flow to the shunt from normal brain capillary (intracerebral blood steal phenomenon). The size and the appearance of initial peak is farely proportional to the clinical course or the results of surgical excision.

This test is extremely atraumatic and is suggested as a screening test or as a means of repeatedly studying the pathophysiology of A-V shunting.

\title{
45. Relative Shunt Flow in Cerebral Arteriovenous Malformations
}

\author{
Keiji Sano, Minoru Jimbo and Isamu Sarto \\ Department of Neurosurgery, Faculty of Medicine, University of Tokyo \\ Teruo NAGAI \\ National Institute of Radiological Sciences
}

\begin{abstract}
Recent advance in neurosurgical treatment of cerebral arteriovenous malformations requires more information about the abnormal hemodynamics of the shunt flow. Although some information can be obtained by a conventional cerebral angiographic technique, the potential value of angiography has not been fully exploited in the quantitative measurement of the shunt flow of the arteriovenous malformations. For the purpose of quantitative measurement of the shunt flow of the arteriovenous malformation, a new radioisotopic technique using $\mathrm{I}^{131}$ labeled macroaggregated human serum albumin (MAA) has been developed.
\end{abstract}

Some dose of $\mathrm{I}^{131}$ MAA was percutaneously injected into the common carotid artery of the patient with arteriovenous malformation on the affected side. Because of its particle size (20-100 micron), some amounts of I $^{131}$ MAA can not pass through the capillary vessels and will lodge in the hemisphere while others transit the arteriovenous anastomosis and will lodge in the capillary vessels of the lungs finally.

After the carotid injection radioactivity of the skull and lungs was measured with the wide angle collimated cintillation detector. The blood flow through the arteriovenous shunt can be calculated from the following equation in terms of percentages on the total blood flow in the one side of the common carotid artery.

$$
\text { relative shunt flow }=\frac{f \times L}{S+f \times L}
$$


where $S$ and $L$ mean the radioactivities of the skull and lungs of the patient after carotid injection of $\mathrm{I}^{131}$ MAA and $f$ is a calibration factor which is obtained from the angiographically normal subject and was calculated as follows:

$$
f=\frac{S_{1}}{L_{2}-L_{1}}
$$

where, $S_{1}$ is radioactivity of the skull after intracarotid injection and $L_{2}-L_{1}$ is the net radioactivities of the lungs as a result of intravenous injection of the same dose.

Seven cases of arteriovenous malformation were investigated by means of this method. Technical details and results were presented and hemodynamic aspects of arteriovenous malformations were discussed in comparison with angiographic findings.

\title{
46. Serial Angiographic Measurement of Rapidity of Cerebral Blood Flow
}

\author{
Toshio Matsubara \\ Neurosurgical Clinic, Okehazama Hospital, Aichi
}

By rapid serial angiographic visualization of cerebral blood vessel, direct measurement of the rapidity of blood flow in each of major cerebral vascular branches may be possible. Author have applied this method to some of clinical cases and showed the normal range of the rapidity calculated on the lateral projection as ollows:

\begin{tabular}{lccccc}
\hline \multicolumn{1}{c}{ part of artery } & $\begin{array}{c}\text { number of } \\
\text { cases }\end{array}$ & maximum-minimum & $\begin{array}{c}\text { average } \\
\text { (in cm./sec.) }\end{array}$ \\
\hline internal carotid & 32 & 36 & - & 48 & 45 \\
anterior and middle cerebral & 32 & 18 & -25 & 20 \\
vertebral-basilar & 8 & 13 & - & 25 & 18 \\
external carotid & 6 & $13-$ & -35 & 20 \\
\hline
\end{tabular}

The wider range of external carotid might be due to greater atmospheric influences, while relatively constant maintenance was observed in internal carotid. Author have taken special considerations on the markedly rapid flow (ca. $90 \mathrm{~cm}$.) sec. in internal carotid-cerebral branches) in parts of arteriovenous malformations which are lacking in capillary resistance, on the contrary, the extraordinary slowing (ca. $20 \mathrm{~cm} . / \mathrm{sec}$. in internal carotid) in some of arteriosclerotic cases which are with increased capillary resistance. Angiospasmus, a functional vascular insufficiency, 